
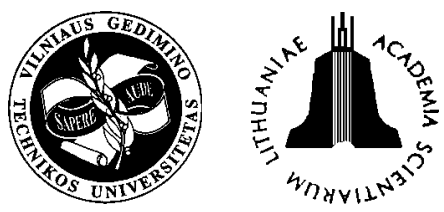

ISSN 1648-4142 TRANSPORT

\title{
QUALITY CONTROL ON THE BASIS OF ENTROPY DETERMINATION
}

\author{
Yasaratne Bandara Dissanayake ${ }^{1}$, Aleksandr Pankov', Vladimir Shestakov ${ }^{3}$ \\ 1,3 Riga Technical University, 1 Kalku,LV-1013 Riga,LLatvia.E-mail: Aviation. Institute@rtu.lv \\ ${ }^{2}$ Transport and Communication Institute,1 Lomonosova, LV-1019 Riga, Latvia. E-mail: pankov@tsi.lv
}

Received 2004-01-06; accepted 2004-02-17

\begin{abstract}
Statistical methods are extensively used in quality control. The method of the application of entropy conception for quality control is proposed. It is based on the use of statistical information about deviations in the operation of a complex system. In the context of this: the structure of the complex system control is considered; the conceptions of controllable and observable systems are introduced. The classification of adverse factor is given influencing upon the system. The example is presented, showing how the application of the proposed method permits to give the evaluation of sources of risks and quality reduction in the complex system.
\end{abstract}

Keywords: statistical methods, entropy, quality, deviations, organization, complex system, quality control.

\section{Introduction}

A complex system of production quality control is the use of statistical methods at all activity cycles of production or service, which is favouring economical growth in the organization functioning to the utmost extent. Developing cyclically, the quality control system passes through certain stages. This cycle is known in the literature as Deming's cycle [1].

The Deming's cycle has always not 1 , but several turns. Each of stages contains a complex of measures that may be formulated in the following form:

\section{P. PLAN}

- Purposes and tasks setting up.

- Determination of methods and means (strategies). Elaboration of plans of measures.

- $\quad$ Teaching and training of specialists.

2. D. DO

- $\quad$ Performing of work.

\section{C. CHECK}

- Control of performed work results.

\section{A. ACTION}

- Realization of control actions. Corrections.

Correct application of contemporary statistical methods at all stages of the Deming's cycle is not restricted to these stages only. They are also applied to market analysis; production designing; definition of reliability, durability, service life etc. specifications.

Specific statistic methods and fields of their application include, but are not limited to: factor analysis, dispersion or regression analysis, risk analysis, criteria of significance evaluation etc.

The evaluation of quality indices deviation significance (danger of production defects) is an important task in the quality control. Correct quantitative determination of potential danger of one or another event (deviation, defect) is permitting to elaborate preventive measures in proper time and direction: the introduction of new or the improvement of existing techniques, personnel training for activity under certain conditions etc. Estimate of significance indicates weak spots in the ensuring of quality. Such evaluation of events is essentially its ranking according to the accepted criterion.

Probabilistic indices are in practice basic criterion of significance degree. These may be both probability itself, obtained using statistical means, and quantity, derived from it, obtainable usually by expert analysis.

In this case, when it fails to obtain statistically meaningful estimate, the task of ranking is restricted to picking out of events priorities according to their significance.

\subsection{Place of numerical methods in the quality control}

There are two main directions of probabilistic quality criteria determination:

- statistical methods;

- methods of expert evaluation.

The first method is permitting to obtain reliable estimate when sufficiently great amount of statistical material is available. The method of expert evaluation is devoid of this shortcoming, but, in its turn, carries in it- 
self an element of subjectivism and depends on the procedure of expert examination making.

Pareto's diagram has taken up widely in the questions of use of quality control numerical methods. It is used for:

- the demonstration of events distribution in general picture in succession of events importance;

- the classification of possibility to improve the quality according to events importance.

Diagram is a simple graphic presentation of events according to a degree of importance from the most frequent events till the least frequent ones [2]. According to Pareto's principle, often only a small amount of events has an influence upon a significant part of action. There fore, when demarcating the most and the least events the most quality improvement will be reached with the least expenditure of resources.

\section{Setting up the problem}

It seems to us that analogous results may be reached using a conception of entropy, $H(x)$. The conception of entropy bears perfectly conventional, formal nature, but it is convenient just for revealing of the most significant events, i.e. for its ranking. Quantitatively $H(x)$ is defined by the following mathematical expressions:

If the probability $P(x i)$ of event (deviation, defect) appearance is calculated for some period of time, then the entropy of system, its uncertainty for the period under consideration will be [3]:

$$
H(x)=\Sigma P(x i) \log P(x i) .
$$

If we have a system, the state of which before exposure to random disturbances (i.e. before observation) was characterized by the probability $P(x i)$ of parameters $(x i)$ values, characterizing this state, but as time passes after the action - by the probability $P k(x i)$, it means that during this time we have obtained the quantity of information that equals to:

$$
J\left(x_{i}\right)=\log _{2}\left[P_{k}\left(x_{i}\right) / P\left(x_{i}\right)\right] .
$$

Connection between successive events $X_{k-1}$ and $X_{k}$ is estimated by correlation functions or conventional entropy. In our case it will be:

$$
H\left(X_{k} / X_{k-1}\right)=\sum_{i=1}^{n} P\left(X_{i}\right) \sum_{j=1}^{m}\left(P ( X _ { j } / X _ { i } ) \operatorname { l o g } \left(P\left(X_{j} / X_{i}\right),\right.\right.
$$

where $m$ is number of states $X_{j}$, connected with appearance of states $X_{i}$;

$\left(P\left(X_{j} / X_{i}\right)\right.$ is the probability of the state $X_{j}$ appearance, provided that the state $X_{i}$ has taken place before it.

In that way, it is possible to forecast quality improvement with the aid of entropy and to evaluate efficiency of quality control measures.

\subsection{General approaches to the elaboration of the quality control system}

Quality control is understood as purposeful activity aimed at revealing, evaluation and elimination of potential deviations, reducing efficiency of a complex system (organization) or use value of production (goods or services) offered by it. From the point of view of control this activity should be based on the information about the state of a complex system (CS), quantitative and qualitative evaluation of various factors influence and forecast for a certain forthcoming period. Thus, the structure of quality control may be presented as a closed system, which includes the elements for gathering, processing and analysis of information about deviations in the operation of various CS subsystems for elaboration and organization of control actions in the form of managerial, organizational, technical and other arrangements. The influence of one or another deviation is defined not only by the degree of possible consequences, but by the frequency of its displays too. Therefore, it is necessary to establish the dependence of quality ensuring system functioning efficiency on the whole complex of possible deviations, taking into account its consequences and frequency of display. When elaborating system of control we'll proceed from the system approach, according to which the system is the aggregate of subsystems and elements, being organized and dynamically and functionally interacting in the process of solution of certain range of tasks (Fig 1).

Here $U(t)$ is vector, characterizing control actions; $Y(t)$ - vector, characterizing output parameters; $X(t)$ vector, characterizing internal state of CS (organization); $V(t)$ - vector of ambient medium action; $R(t)$ - index, characterizing presence of internal binary relationships between elements being inside of organization.

In general any CS control system may be presented in the form of a structure consisting of three blocks (Fig 2):

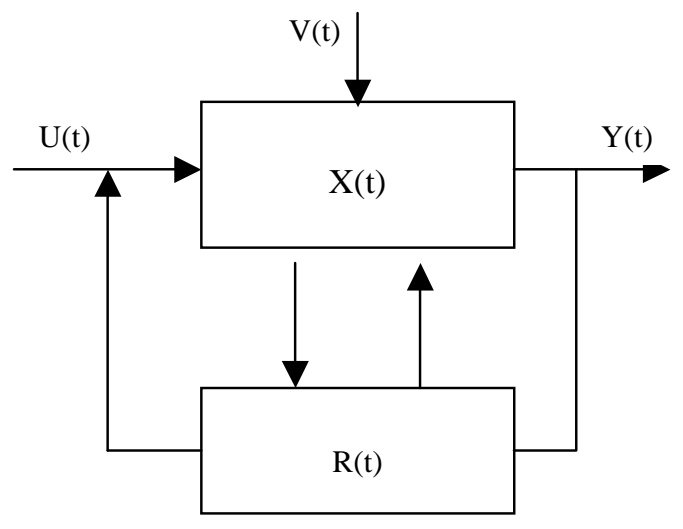

Fig 1 


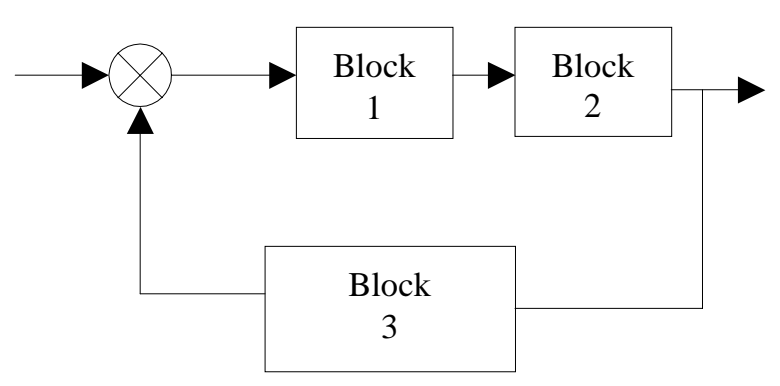

Fig 2

The first block, the unit of information presentation is performing the functions of information collection, presentation, processing and storage, substantiation of taken decisions and so on.

The second block, the unit of control measures elaboration is performing the functions of requirements, regulations, instructions and so on working on the basis of standards and other regulating documents.

The third block, the unit of measure fulfillment is performing the functions of worked out measure fulfillment checking, efficiency examination, elaboration of correcting actions and its insertion into the control system.

The current state of a complex system is formed by multitude of states of all elements $X(t)$ of the system, fixed at some moment of time $t$. The state of CS as a dynamical system at the moment $t$ is described by an aggregate of physical variables (parameters):

$$
X 1(t), X 2(t), \ldots, X n(t),
$$

that are vectors (degrees of freedom) of the system states:

$$
X(t)=\{X 1(t), X 2(t), . . X n(t)\}
$$

Knowing initial values of variables: $X 1(t 0), X 2(t 0)$, ..., $X n(t 0)$, it is possible to transfer the system, changing input signal of the signal (control signal) $U(t)$, to the new state with parameters:

$$
X 1(t), X 2(t), \ldots, X n(t),
$$

i.e. to realize control of system. From the other side, knowing values of the input signal $U(t)$ and output signal $Y(t)$ during sizable time interval, we can determine the initial (starting) state of system:

$$
\{X 1(t 0), X 2(t 0), \ldots, X n(t 0)\},
$$

and its current state: $\{X 1(t), X 2(t), \ldots, X n(t)\}$, i.e. to subject it to observation. In this connection let us introduce conceptions: controllable system (subsystem) and observable system (subsystem).

Controllable system is such CS, when for any points at time $t 0$ and $t 1$, at $t 1>t 0$ and any preset states $X 0$ and $X 1$, there exists control action $U(t)$ in the interval $t 0<$ $t<t 1$ that transfers initial state $X 0$ of the system to final state $X 1$.

Observable system is $\mathrm{CS}$, for which its initial state
$X 0$ and current state $X(t)$ may be unequivocally determined on evidence derived from measurement or observation of vectors $U(t)$ and $Y(t)$ on finite time interval $t 0<$ $t<t 1$.

Starting from the said it may be thought that it is possible to separate out in the complex system (to divide up the system to) four types of subsystems:

- controllable and observable $-\mathrm{S}_{\mathrm{co}}$;

- controllable and nonobservable - $\mathrm{S}_{\mathrm{cno}}$;

- noncontrollable and nonobservable $-\mathrm{S}_{\text {ncno }}$;

- noncontrollable and observable $-\mathrm{S}_{\text {nco }}$.

For CS to be controllable and observable it should consist of subsystems of $\mathrm{S}_{\mathrm{co}}$ type only. The analysis of the CS state may be carried out only in the case when it has no subsystems of $\mathrm{S}_{\text {cno }}$ and $\mathrm{S}_{\text {ncno }}$ types, but consists of subsystems of $\mathrm{S}_{\text {co }}$ and $\mathrm{S}_{\text {nco }}$ only.

If the subsystem is nonobservable $\left(\mathrm{S}_{\mathrm{cno}}\right)$, it cannot influence upon the subsystems $\mathrm{S}_{\text {co }}$ and $\mathrm{S}_{\text {nco }}$ since these are observable.

If the subsystem is noncontrollable and nonobservable $\left(\mathrm{S}_{\mathrm{ncno}}\right)$, it cannot be influenced upon by the controllable subsystems $\mathrm{S}_{\text {co }}$ and $\mathrm{S}_{\text {cno }}$.

The observability of the systems is understood as the presence, the possibility to fix at different points of time quantitative or qualitative indices (deviations), according to which current state $\{X 1(t), X 2(t), \ldots, X n(t)\}$ of the system as a whole and its subsystems (elements) in particular may be inferred. The property of observability permits to carry out analysis, to evaluate quality, so to make decisions, to realize forecasts, to select control actions etc.

The uncertainty of the system is the absence of clearcut notion of the system state (i.e. its parameters $\{X 1(t)$, $X 2(t), \ldots, X n(t)\})$ which, in its turn, is determined by the presence of certain amount of information.

So, if the actions of different kinds (control actions $U(t)$ or factors of ambient medium $V(t)$ ) act on the system at random points of time, its state $X(t)$ begins to change and the uncertainty will take place till the state that is final becomes settled, becomes formed. If some system observation is carried out in the process, i.e. some parameters are fixed, the uncertainty degree reduces or eliminates.

As it has been noted above, the measure of CS uncertainty is characterized by entropy $H(x)$. But since the uncertainty depends on informativeness (amount of information), then the entropy is a measure of amount of information during the system state observations.

The efficiency of quality control in many cases is determined by the timeliness of unexpected deviations revealing and eliminating. Therefore basic principles of control are:

- timely revealing of deviations that can lead to loss of quality;

- $\quad$ evaluation of its degree of danger; 
- forecast of trends of influence of various factors upon quality;

- $\quad$ timely working out of preventive arrangements;

- realization of operative and long-term measures for non-admission or restriction of deviations that can lead to loss of quality;

- checking of control measures efficiency.

The efficiency of operative quality control is determined, first of all, by a possibility of timely revealing of dangerous deviations and making decisions for its elimination and localization.

Periodic analyses of quality indices are the main source of information for elaboration of long-term (strategic) control actions. The efficiency of long-term control actions in many cases depends on the organization of these analyses, their completeness and objectivity.

The analysis and forecast of the influence of factors throughout all statistical information for several years permit to define the main effective directions of preventive activity.

\section{Use of entropy for quality control}

Quality is negatively affected upon by a great number of factors. Let us name them adverse factors.

As this takes place each adverse factor should be understood as any action or inaction, case, condition, circumstance, something else.

Its presence or, contrary, absence changes the probability of appearance of deviations from established quality indices.

Variety and ramifiedness of CS (organization) and its subsystems stipulate the display of abundance of adverse factors, classification of which may be performed with different degree of working out in details and with different dividing according to subject headings, in other words with a different degree of generalization. It includes:

- unsatisfactory general management of organization;

- unsatisfactory organization of work;

- low professional level of personnel;

- psychophysiologic state (displays) in collective;

- low level of discipline;

- design and production shortcomings of equipment in use;

- unsatisfactory maintenance of equipment in use;

- unsatisfactory provision of all kinds of functioning;

- external active actions;

- unestablished reasons.

These factors may be considered as generalized. It is possible to separate out from each of them a whole group of particular, more concrete factors, which may be fixed as a result of observation.
So, for the first group we'll have:

- a low level of documentation, regulating functioning of the organization;

- unsatisfactory work quota setting of the personnel and its admission to work etc.

For the second group:

- violation of normative activity by the personnel;

- unsatisfactory forming of sub-units and organization of its work;

- insufficiently clear-cut planning and checking of activities etc.

The following may be related to a low professional level of the personnel:

- low standard of management at all levels;

- unsatisfactory training of the personnel for work in given circumstances etc.

Psychophysiological state (displays) in the collective is characterized by:

- sudden falling ill;

- physical trauma;

- psychophysiological stress;

- overwork;

- alcoholic and narcotic intoxication;

- poisoning;

- unfavourable biorhythmic factors etc.

The level of personnel discipline is of particular importance. Group of design and production factors means production imperfections of the equipment in use.

The unsatisfactory maintenance of the equipment is stipulated by unsatisfactory provision and organizing of operation, routine repairs, a low professional level of specialists.

Unsatisfactory provision includes all kinds of provision: technical, financial, information and so on.

External active actions are actions from external medium.

All these factors may be considered as special and in practical activity be determined quantitatively, i.e. these are the factors that make the organization observable.

\subsection{Connection between special and generalized} factors

Several measured random quantities referred to as special factors (SF) will be considered as closely interconnected by some other random quantity, let us name it a generalized factor (GF). The degree of generalization or, on the contrary, breaking up into smaller units, has no restrictions of fundamental nature.

All depends on research purposes, considered system (subsystem) or its separate links. This way, it is generally accepted to divide all adverse factors into three groups:

- human factors,

- technical factors,

- adverse factors of external medium. 
It is the highest level of factors generalization, though this classification is sufficiently conventional too, because in many cases it is sufficiently difficult to separate out a technical factor from a human factor, and vice versa. As any technique is a product of human intellect and work, then all shortcomings of its designing and production may also be related to the human factor.

The problem arises, is it possible to separate out from measured special factors generalized, i.e. explaining observed connections, factor, unamenable to direct measurement. Special factor is quantitative description of random occurrence, its value depends on outcome of the random occurrence. Starting prerequisite of factor analysis is the presence of interrelation between several simultaneously observed quantities, special factors.

In quality control questions the generalized factors express measures that are necessary for quality improvement. Using the conception of entropy it is possible to rank generalized factors according to the degree of their significance for quality improvement [4].

Let us demonstrate this with an example.

\section{Example and conclusions}

As a result of one year long study and the analysis of some company filling stations work six deviations (special factors) $X i(i=1 \ldots 6)$ in its work quality have been revealed:

$X 1$ - non-fulfillment of oral or written instructions, if any;

$X 2$ - inopportune (non-qualitative) analysis of fuels and lubricants;

$X 3$ - transport means filling up with fuels and lubricants, that do not correspond to standards;

$X 4$ - violation of filling up rules;

$X 5$ - filling up with fuels and lubricants with not corresponding grades;

$X 6$ - violation of fuels and lubricants transportation rules; frequency of its display (probability) is determined.

Using logical reasoning, one can establish that all these special factors may be united into two generalized factors, A and B.

Factor A is closely connected with special factors $X 1, X 2, X 3$, and Factor B - with factors $X 3, X 4, X 5, X 6$. Graphically it will be in the form, shown in Fig 3.

Generalized factors $\mathbf{A}$ and $\mathbf{B}$ may be formulated as:

A - checking of fuels and lubricants quality;

B - carrying out of direct filling up of transport means.

Factors A and B are unamenable to direct measurement and may be determined only as a result of the system observation during a certain period and the analysis of obtained results of observation (determination of display frequency).

Now it is necessary to place them according to pri-

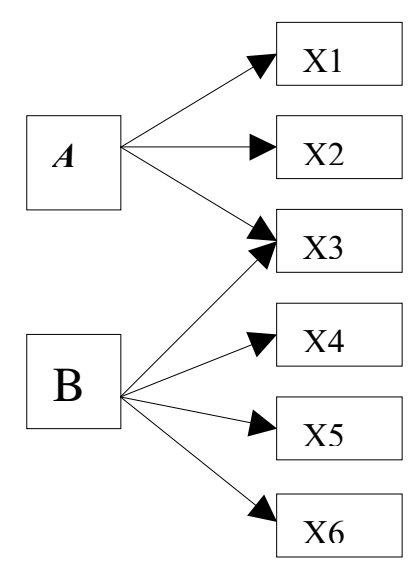

Fig 3

orities. This may be done on the basis of entropy value determination for each of these two factors.

The method of analysis is required to be so that the reflection and reproducing of real, existing dependences shall be sufficiently precise. Entropy method may be one of such methods. Its essence is as follows.

Carrying out observations for a certain period of time and fixing special factors, we can obtain magnitudes of probabilities $P(x i)$ of its display for this period. Using this data according to formula (1), we determine the entropy magnitudes for factors A and B. So, in this case for fuels and lubricants service it is established by calculation according to formula:

$$
\mathbf{H}(\mathbf{A})=-4,2854 ; \quad \mathbf{H}(\mathbf{B})=-2,5674 \text {. }
$$

It means that in a given company all risks in its functioning and loss of quality are stipulated mainly by the absence of appropriate checking of fuels and lubricants quality.

It means that the main attention of a manager should be paid to solving of these questions. Their solving requires elaboration, realization and checking of the efficiency of control measures. In our diagram of control, Fig 2, it is block 2. One can continue this example and evaluate, according to formulae (2) and (3), the level of connection between controlling arrangements and their efficiency and so on.

\section{References}

1. Kruglova M. G. and others. Management of qualitative systems (Менеджмент систем качества). Moscow: Izdatelstvo standartov, 1997 (in Russian).

2. International standard ISO 94004-4, 2000.

3. Sakach and others. Flight safety (Безопасность полетов). Moscow: Transport, 1987 (in Russian).

4. Jaroslavceva J. Cilvēciskā faktora vadība lidojumu inženiertehniskā nodrošinājuma sistēmā. In: Scientific proceedings of Riga Technical University, Transport and Engineering, series 6, Riga, 2002. 